\title{
Assessment of the reproductive toxicology of bromochlorodifluoromethane (BCF, halon 1211) in the rat
}

\author{
G A de S WICKRAMARATNE, D J TINSTON, DIANE L KINSEY, J E DOE \\ From Imperial Chemical Industries plc, Central Toxicology Laboratory, Alderley Park, Macclesfield, Cheshire, \\ $U K$
}

\begin{abstract}
The effect of bromochlorodifluoromethane (BCF) on reproduction in the rat has been investigated in two studies. Pregnant female rats were exposed by inhalation to 1000,10000 , or $50000 \mathrm{ppm} \mathrm{BCF}$ for six hours a day on days six to 15 of gestation (day of mating = day 0 ). Exposure to $50000 \mathrm{ppm}$ BCF caused a reduction in maternal weight gain over the exposure period but there was no evidence of either teratogenicity or embryo/fetotoxicity at any concentration. In a study designed to assess the potential effect of BCF during a complete reproductive cycle male and female rats were exposed to $5000 \mathrm{ppm}$ or $25000 \mathrm{ppm}$ BCF for six hours a day for five days a week for 10 weeks (males) or three weeks (females) before mating. Exposure to BCF continued during mating and up to day 20 of gestation for half the females which were subsequently allowed to litter and the development of their offspring monitored. The remaining females were removed from exposure to BCF after mating and killed on day 20 of gestation for examination of their uterine contents. There were no effects on adult fertility, pup numbers, survival, or pup development. It was concluded that $\mathrm{BCF}$ had no reproductive toxicity potential in the rat.
\end{abstract}

Halogenated fire extinguishants have been used extensively since the early 1920 s when carbon tetrachloride was used for this purpose. Since the 1950s, materials that are less toxic than carbon tetrachloride have been introduced, one being bromochlorodifluoromethane (BCF, halon 1211). BCF is much less toxic than carbon tetrachloride, its major activity being effects on the central nervous system caused by exposure to high concentrations. 'In addition to assessing the toxicity of brief exposures such as may be experienced during fire fighting it is important to assess the possible hazards to those employed in the manufacture of $\mathrm{BCF}$ and the filling of fire extinguishers. As part of this evaluation, the effect of BCF on embryonic development and reproduction in the rat has been examined.

\section{Materials and methods}

TEST MATERIAL

BCF was supplied by ICI Chemicals and Polymers Group as bromochlorodifluoromethane (BCF). The sample conformed to plant specification being $>94 \%$ BCF with water as the major impurity, c 5\%.

\section{ANIMALS}

Rats of the Alpk:AP (Wistar derived) strain were supplied from the animal breeding unit, Alderley Park. For the teratology study, female rats weighing between 190 and $320 \mathrm{~g}$ (about 9-14 weeks old) were mated overnight and the following morning vaginal smears were taken for confirmation of mating. The day on which spermatozoa were detected in vaginal smears was designated day 0 of pregnancy.

For the reproduction study, young adult male and female rats which were sexually mature at the start of the exposure period were used (males eight weeks old 200-290 g, females seven weeks old 120-170 g).

\section{EXPOSURE CHAMBERS}

The chambers had an internal volume of $3.4 \mathrm{~m}^{3}$ and were constructed of stainless steel. ${ }^{2}$ Air entered at the front of each chamber and was extracted at the back. Within each chamber were six cage levels and excreta trays that rotated at a rate of 0.5 revolutions a minute. The air flow through each chamber was $300 \mathrm{l} / \mathrm{min}$ monitored by Rotameter. The air was conditioned to $22^{\circ} \mathrm{C}$ and $55 \%$ relative humidity monitored by wet and dry bulb thermometers. 
ANIMAL HUSBANDRY

The rats were maintained in wire mesh cages housed within the exposure chambers throughout the exposure phases of the studies and supplied with food (PCD, BP Nutrition Ltd) and water ad libitum except during exposures. At other times the rats were maintained in wire mesh cages on racks and supplied with food and water ad libitum. Female rats were housed in cages with solid bottoms and paper bedding before littering where appropriate.

\section{ATMOSPHERE GENERATION AND ANALYSIS}

Atmospheres were generated by metering appropriate amounts of BCF into the input air of each chamber using calibrated flowmeters.

The concentrations of BCF in each chamber were analysed at least once an hour through a sampling point in the back of the chamber connected to a gas chromatograph or a Miran infra-red analyser by copper tubing and a multipoint sampler.

\section{STUDY DESIGNS}

Teratology study

On days 6-15 inclusive of gestation, groups of 24 pregnant female rats were exposed to concentrations of BCF of $0,1000,10000$, or 50000 ppm for six hours a day. The body weight of each rat was recorded on day 0 , daily on days 5-15, and on day 20 . Food consumption was monitored on day 2 , daily on days 6-16, and on day 20. Each animal was observed daily to monitor its clinical condition.

On day 20 of gestation each rat was killed by overexposure to halothane BP vapour and the uterus was immediately dissected out and the weight recorded. The number of corpora lutea in each ovary was recorded. The uterus was opened and the number of implantations, early, late, and fetal deaths was recorded. Each live fetus was removed from its uterine attachment, freed of fetal membranes, the umbilical cord was cut, and the fetus weighed. Each fetus was examined externally for gross abnormalities.Alternate fetuses in each litter were fixed in $70 \%$ alcohol for subsequent evisceration (during which the sex was determined and the abdominal and thoracic contents were examined for abnormalities) and staining with Alizarin Red S for examination of the osseous skeleton under a stereomicroscope. The remaining fetuses were fixed in Bouin's fluid and transferred to $70 \%$ alcohol. Each was then sectioned at approximately $2 \mathrm{~mm}$ intervals and the sections examined under a stereomicroscope. Any abnormalities were noted and the sex of the fetus determined.

\section{Reproduction study}

Groups of 12 male and 24 female rats were assigned to two exposure groups (5000 and 25000 ppm BCF) or one control group. The rats were exposed to the appropriate concentrations of BCF for six hours a day, five days a week, the males being exposed for 10 weeks and the females for two weeks before the pairing period. The rats were observed daily to assess their clinical condition and their body weight and food consumption were measured weekly.

After the last exposure of the pre-pairing period, each male was housed with two female rats and the rats were exposed to the appropriate concentration of BCF for six hours a day for a maximum of two weeks. Each female rat was examined daily using a vaginal swab, and the preparation of a smear on a microscope slide, until successful mating occurred as shown by either a vaginal plug or the presence of sperm.

The first female rat, in each cage, which showed evidence of successful mating was allocated to subgroup A, housed separately, and continued to be exposed to BCF until day 20 of gestation. The rats in subgroup A were then transferred to cages with solid bottoms and allowed to litter. Any rat not producing a litter by day 24 post coitum was killed and subjected to a postmortem examination to determine if the rat had been pregnant. The following observations were made on the dams and their litters:

date of parturition and duration of pregnancy; number of male and female pups born alive or dead in each litter;

number of pups alive or dead daily for 28 days; weight gain, detailed observations, and developmental parameters (unfolding of pinna, hair growth, eye opening) recorded on an individual pup basis;

weight gain and clinical condition of each dam for 28 days.

The dams and pups were killed after 28 days but were not subjected to a postmortem examination.

The second female rat to show evidence of successful mating was assigned to subgroup B. These rats were removed from the exposure chamber and housed singly until day 20 of gestation, when they were killed and subjected to a gross postmortem examination. The uterine contents were examined and the numbers of live and dead fetuses and any resorptions were recorded.

\section{Results}

\section{TERATOLOGY STUDY}

Atmosphere analysis

The daily mean concentrations of BCF were within $\pm 15 \%$ of all three target concentrations to give overall means of: $1000 \mathrm{ppm}:-1021 \pm 64 \mathrm{ppm}, 10000$ ppm:-10059 \pm 459 ppm, 50000 ppm:- $48343 \pm$ $2532 \mathrm{ppm}$. No BCF was detected in the control chamber. 
Table 1 BCF teratology study: pregnancy performance. (Standard deviations in parentheses)

\begin{tabular}{|c|c|c|c|c|}
\hline & \multicolumn{4}{|c|}{ Exposure concentrations of BCF in air (ppm) } \\
\hline & 0 & 1000 & 10000 & 50000 \\
\hline $\begin{array}{l}\text { No of rats mated } \\
\text { No of pregnancies } \\
\text { No of abortions } \\
\text { No of maternal deaths } \\
\text { Total corpora lutea } \\
\text { Total implantations } \\
\text { Live implantations } \\
\text { Male fetuses } \\
\text { Female fetuses } \\
\text { Total dead implantations } \\
\text { Early deaths } \\
\text { Late deaths } \\
\text { Fetal weight (g) }\end{array}$ & $\begin{array}{r}24 \\
24 \\
0 \\
0 \\
340 \\
312 \\
304 \\
149 \\
155 \\
8 \\
6 \\
2 \\
3 \cdot 71(0 \cdot 27)\end{array}$ & $\begin{array}{l}24 \\
23 \\
0 \\
0 \\
300 \\
282 \\
269 \\
136 \\
133 \\
13 \\
11 \\
2 \\
3 \cdot 70(0 \cdot 28)\end{array}$ & $\begin{array}{l}24 \\
23 \\
0 \\
0 \\
321 \\
300 \\
292 \\
140^{*} \\
151^{*} \\
7 \\
6 \\
1 \\
3 \cdot 56(0 \cdot 17) \dagger\end{array}$ & $\begin{array}{r}24 \\
24 \\
0 \\
0 \\
326 \\
299 \\
284 \\
137 \\
147 \\
15 \\
13 \\
2 \\
3.65(0 \cdot 23)\end{array}$ \\
\hline $\begin{array}{l}\text { Pregnancy frequency } \\
\text { Mean gravid uterus weight } \\
\text { Corpora lutea/pregnancy } \\
\text { Live implantations/pregnancy } \\
\text { Early deaths/pregnancy } \\
\text { Late deaths/pregnancy } \\
\text { Sex ratio (male:female) }\end{array}$ & $\begin{array}{l}100 \% \\
75 \cdot 1(12 \cdot 3) \\
14 \cdot 2(2 \cdot 1) \\
12 \cdot 7(2 \cdot 1) \\
0 \cdot 25(0 \cdot 4) \\
0.08(0 \cdot 3) \\
0.96\end{array}$ & $\begin{array}{l}95 \cdot 8 \% \\
68 \cdot 8(11 \cdot 9) \\
13.0(2.0) \\
11.7(2.3) \\
0.5(0.6) \\
0.09(0.3) \\
1.02\end{array}$ & $\begin{array}{l}95.8 \% \\
71.9(10.3) \\
13.9(1.9) \\
12.7(1.9) \\
0.3(0.5) \\
0.04(0.2) \\
0.92\end{array}$ & $\begin{array}{l}100 \% \\
68.5(8.9) \\
13.5(1.6) \\
11.8(1.6) \\
0.5(0 \cdot 6) \\
0.08(0.3) \\
0.93\end{array}$ \\
\hline
\end{tabular}

*One fetus not sexed.

†Statistically different from controls $\mathrm{p}>0.05$ (Student's $t$ test).

\section{Maternal clinical condition}

There were no changes in clinical condition which could be attributed to exposure to BCF, although the weight gain of the rats exposed to $50000 \mathrm{ppm}$ was statistically significantly reduced during days 5-15 (controls: $45 \pm 10 \mathrm{~g}, 1000$ ppm: $43 \pm 8 \mathrm{~g}, 10000$ ppm: $43 \pm 9 \mathrm{~g}, 50000 \mathrm{ppm}: 33 \pm 8 \mathrm{~g}$ ). The food consumption of the rats exposed to $50000 \mathrm{ppm}$ was also reduced during this period. There was no evidence for an effect on the rats exposed to 1000 or $10000 \mathrm{ppm}$ BCF.

\section{Litter data}

As shown in table 1 there were no effects of exposure to BCF on pregnancy performance. There was a statistically significant reduction in fetal weight in the $10000 \mathrm{ppm}$ group which, in the absence of an effect in the $50000 \mathrm{ppm}$ group, is unlikely to be related to treatment.

\section{Skeletal and visceral data}

Table 2 shows the results of the skeletal assessment. There were no effects attributable to exposure to BCF. The visceral examination (table 3) also showed no evidence of an effect of BCF.

\section{REPRODUCTION STUDY}

Atmosphere analysis

The daily mean concentrations were all within $15 \%$ of the target concentrations thoughout the study, except for two days when the $25000 \mathrm{ppm}$ target group were exposed to $29000 \mathrm{ppm}$ BCF. There was no BCF detected in the control chamber.

\section{Pre-pairing period observations}

There were no treatment related effects on bodyweight gain or clinical condition except for a reduction in bodyweight gain during weeks 1 and 2 in the male rats exposed to $25000 \mathrm{ppm} \mathrm{BCF}$.

\section{Pairing period observations}

The rats appeared clinically normal throughout the pairing period. Positive signs of mating appeared to have occurred slightly earlier in the rats exposed to 25000 ppm BCF, but this observation is of dubious significance. The incidences of coitus and pregnancy in each female rat were evaluated as positive smear and pregnant, positive smear and not pregnant, negative smear and pregnant, and negative smear and not pregnant. Examination of the data using Fisher's exact test (one sided) showed no significant differences between the controls and either of the treated groups.

\section{Post-pairing and postpartum period (subgroup A)}

There were no effects attributable to BCF during the exposures for the first 20 days of gestation and all the rats appeared clinically normal. The duration of pregnancy did not differ between either of the groups exposed to BCF and the controls.

The group mean values for the number of male and female pups in each litter and the sex ratio for day 1 (day of birth) and day 27 are shown in table 4, which shows no differences between the test and control groups. The lactation index (number of pups alive at day 28/number of pups alive at day 1 ) and mortality data for the pups, as shown in table 5 , also showed no differences between the test and control animals. 
Table 2 BCF teratology study: skeletal variants

\begin{tabular}{|c|c|c|c|c|c|c|c|c|}
\hline \multirow[b]{3}{*}{ Variants } & \multicolumn{8}{|c|}{ Exposure concentrations of BCF in air (ppm) } \\
\hline & \multicolumn{2}{|l|}{0} & \multicolumn{2}{|l|}{1000} & \multicolumn{2}{|c|}{10000} & \multicolumn{2}{|c|}{50000} \\
\hline & No & $\%$ & No & $\%$ & No & $\%$ & No & $\%$ \\
\hline \multirow{8}{*}{$\begin{array}{l}\text { Skull partially ossified } \\
\text { Palate partially ossified } \\
\text { Frontal partially ossified } \\
\text { Parietal partially ossified } \\
\text { Interparietal partially ossified } \\
\text { Skull wide fontanelle } \\
14 \text { thoracic vertebrae } \\
1 \text { or more thoracic centra bipartite or } \\
\quad \text { partially ossified }\end{array}$} & 1 & 0.6 & \multicolumn{2}{|l|}{0} & 3 & $2 \cdot 0$ & \multicolumn{2}{|l|}{0} \\
\hline & $\mathrm{i}$ & 0.6 & \multirow{2}{*}{\multicolumn{2}{|c|}{$\begin{array}{l}0 \\
0\end{array}$}} & \multicolumn{2}{|l|}{0} & \multicolumn{2}{|l|}{0} \\
\hline & 1 & 0.6 & & & $2 \cdot 7$ & $2 \cdot 7$ & \multicolumn{2}{|l|}{0} \\
\hline & 9 & $5 \cdot 8$ & \multicolumn{2}{|r|}{2.2} & \multicolumn{2}{|r|}{$4 \cdot 0$} & \multirow{2}{*}{\multicolumn{2}{|c|}{$\begin{array}{l}6 \cdot 2 \\
5 \cdot 5\end{array}$}} \\
\hline & 8 & $5 \cdot 1$ & 3 & $2 \cdot 2$ & 7 & $4 \cdot 7$ & & $5 \cdot 5$ \\
\hline & 14 & $9 \cdot 0$ & 7 & $5 \cdot 1$ & 19 & $12 \cdot 7$ & 7 & $4 \cdot 8$ \\
\hline & 1 & 0.6 & 0 & & 3 & $2 \cdot 0$ & 1 & 0.7 \\
\hline & 6 & 3.8 & 5 & 3.6 & 6 & $\mathbf{4 \cdot 0}$ & 5 & $3 \cdot 4$ \\
\hline 5 lumbar vertebrae & 1 & 0.6 & 0 & & 3 & 2.0 & 0 & 34 \\
\hline 4 or more caudal centra ossified & 118 & $75 \cdot 6$ & 118 & $86 \cdot 1$ & 116 & $77 \cdot 3$ & 118 & $81 \cdot 4$ \\
\hline $\begin{array}{l}3 \text { or more caudal arches ossified } \\
\text { Ribs: }\end{array}$ & 1 & 0.6 & 3 & $2 \cdot 2$ & 0 & & 3 & $2 \cdot 1$ \\
\hline $\begin{array}{l}\text { Ribs: } \\
\quad 12 / 12\end{array}$ & 1 & 0.6 & 0 & & 0 & & 0 & \\
\hline $13 / 12$ & 0 & & 1 & 0.7 & 0 & & 0 & \\
\hline $12 / 13$ & 0 & & 2 & $1 \cdot 5$ & 1 & 0.7 & 0 & \\
\hline $14 / 13$ & 0 & & 0 & & 0 & & 0 & \\
\hline $13 / 14^{v}$ & 6 & 3.6 & 5 & $3 \cdot 6$ & 6 & $4 \cdot 0$ & 2 & 1.4 \\
\hline $14^{v^{\prime}} / 13$ & 9 & $5 \cdot 8$ & 1 & 0.7 & 5 & $3 \cdot 3$ & 8 & $5 \cdot 5$ \\
\hline $14^{v} / 14$ & 1 & 0.6 & 0 & & 1 & 0.7 & 0 & \\
\hline $14 / 14^{v}$ & 0 & & 0 & & 2 & & 0 & \\
\hline $14^{v / 14^{v}}$ & 8 & $5 \cdot 1$ & 14 & $10 \cdot 2$ & 10 & $6 \cdot 7$ & 9 & $6 \cdot 2$ \\
\hline Partially ossified sternebrae: & & & & & & & & \\
\hline No 1 & 4 & $2 \cdot 6$ & 1 & 0.7 & 1 & 0.7 & 3 & $2 \cdot 1$ \\
\hline 2 & 17 & 10.9 & 24 & $17 \cdot 5$ & 32 & $21 \cdot 3$ & 27 & $18 \cdot 6$ \\
\hline 3 & 6 & 3.8 & 7 & $5 \cdot 1$ & 7 & 4.7 & 9 & 6.2 \\
\hline 4 & 24 & $15 \cdot 4$ & 13 & $9 \cdot 5$ & 21 & 14.0 & 25 & $17 \cdot 2$ \\
\hline 5 & 97 & $62 \cdot 2$ & 91 & $66 \cdot 4$ & 108 & $72 \cdot 0$ & 100 & $69 \cdot 0$ \\
\hline 6 & 38 & $24 \cdot 4$ & 27 & $19 \cdot 7$ & 39 & $26 \cdot 0$ & 30 & $20 \cdot 7$ \\
\hline Unossified sternebrae: & & & & & & & & \\
\hline No 1 & 1 & 0.6 & 0 & & 2 & $1 \cdot 3$ & 2 & 1.4 \\
\hline 2 & 1 & 0.6 & 0 & & 1 & 0.7 & 0 & \\
\hline 3 & 1 & 0.6 & 0 & & 4 & $2 \cdot 7$ & 0 & \\
\hline 4 & 4 & 0 & 0 & & $\mathrm{i}$ & 0.7 & 0 & \\
\hline 5 & 12 & $7 \cdot 7$ & 10 & $7 \cdot 3$ & 16 & $10 \cdot 7$ & 20 & $13 \cdot 8$ \\
\hline 6 & 3 & 1.9 & 3 & $2 \cdot 2$ & 8 & $5 \cdot 3$ & 7 & $4 \cdot 8$ \\
\hline 1 or more sternebrae misaligned & 29 & $18 \cdot 6$ & 15 & 10.9 & 22 & $14 \cdot 7$ & 10 & 6.9 \\
\hline 1 or more sternebrae bipartite & 15 & $9 \cdot 6$ & 11 & $8 \cdot 0$ & 16 & $10 \cdot 7$ & 15 & $10 \cdot 3$ \\
\hline Mean No of partially ossified sternebrae & & $1 \cdot 19$ & & $1 \cdot 19$ & & $1 \cdot 39$ & & $1 \cdot 40$ \\
\hline $\begin{array}{l}\text { Mean No of partially unossitied } \\
\text { sternebrae }\end{array}$ & & $0 \cdot 12$ & & 0.09 & & $0 \cdot 19$ & & 0.19 \\
\hline Ossification grade:* & & & & & & & & \\
\hline Manus: 3 & 1 & 0.6 & 3 & $2 \cdot 2$ & 3 & $2 \cdot 0$ & 0 & 0 \\
\hline 4 & 39 & $25 \cdot 0$ & 28 & $20 \cdot 4$ & 39 & $26 \cdot 0$ & 44 & $30 \cdot 3$ \\
\hline 5 & 57 & $36 \cdot 5$ & 32 & 23.4 & 42 & $28 \cdot 0$ & 35 & $\begin{array}{l}24.1 \\
25.5\end{array}$ \\
\hline 6 & 22 & $14 \cdot 1$ & 47 & $34 \cdot 3$ & 38 & $25 \cdot 3$ & 37 & $25 \cdot 5$ \\
\hline 7 & 37 & $23 \cdot 7$ & 27 & 19.7 & 28 & 18.7 & 29 & 20.0 \\
\hline Pes: & 2 & $1 \cdot 3$ & 5 & 3.6 & 7 & $4 \cdot 7$ & 3 & $2 \cdot 1$ \\
\hline 4 & 90 & $57 \cdot 7$ & 61 & 44.5 & 79 & 52.7 & 72 & $49 \cdot 7$ \\
\hline 5 & 19 & $12 \cdot 2$ & 33 & $24 \cdot 1$ & 20 & $13 \cdot 3$ & 29 & $20 \cdot 0$ \\
\hline 6 & 0 & 0 & 4 & 2.9 & 5 & $3 \cdot 3$ & 8 & $5 \cdot 5$ \\
\hline 7 & 45 & $28 \cdot 8$ & 34 & $24 \cdot 8$ & 39 & $26 \cdot 0$ & 33 & $22 \cdot 8$ \\
\hline No of fetuses examined & 156 & & 137 & & 150 & & 145 & \\
\hline No of litters examined & 24 & & 23 & & 23 & & 24 & \\
\hline
\end{tabular}

V, vestigial rib.

*Ossification grade of the manus and pes is based on a 10 point scale where each bone is assessed. The lower the score the greater the degree of ossification.

There were no differences in the weight gain of the pups over the postpartum period. There were no differences in the developmental parameters, table 6, except that pinna detachment occurred statistically significantly earlier in the rats whose parents had been exposed to $25000 \mathrm{ppm} \mathrm{BCF}$.
Uterine observations (subgroup B)

The rats in subgroup $B$ showed no differences between the treated and control groups in either bodyweight gain or clinical condition after their removal from the exposure chambers on day 0 of pregnancy. The observations made at the postmortem examinations 
Table 3 BCF teratology study: visceral examination

\begin{tabular}{|c|c|c|c|c|}
\hline \multirow[b]{2}{*}{ Variants } & \multicolumn{4}{|c|}{$\begin{array}{l}\text { Exposure concentrations of BCF in air } \\
(\text { ppm })\end{array}$} \\
\hline & 0 & 1000 & 10000 & 50000 \\
\hline \multicolumn{5}{|l|}{ Pelvic dilatation: } \\
\hline Left & 8 & 4 & 3 & 5 \\
\hline Right & 6 & 4 & 10 & 4 \\
\hline Bilateral & 14 & 8 & 4 & 6 \\
\hline \multicolumn{5}{|l|}{ Lateral ventricle enlarged: } \\
\hline Left & 0 & 1 & 0 & 0 \\
\hline Bilateral & 8 & 11 & 12 & 12 \\
\hline \multicolumn{5}{|l|}{ Cheek pouch enlarged: } \\
\hline Left & 0 & 0 & 0 & 0 \\
\hline Right & 0 & 0 & 1 & 0 \\
\hline \multicolumn{5}{|l|}{ Kidney displaced: } \\
\hline Posteriorly & 1 & 0 & 0 & 0 \\
\hline \multirow{3}{*}{$\begin{array}{l}\text { Left } \\
\text { Vestigial tail and multiple } \\
\text { abnormalities }\end{array}$} & & & & \\
\hline & 1 & 0 & 0 & 0 \\
\hline & 1 & 0 & 0 & 0 \\
\hline No examined & 148 & 132 & 142 & 139 \\
\hline
\end{tabular}

are shown in table 7 . There was a slight, although statistically significant, reduction in the number of fetuses in the rats which had been exposed to 25000 ppm BCF. There were no differences in the gross appearance of either the dams or the fetuses.
Table 6 BCF reproduction study: pup development parameters. (Values are means \pm standard deviation with number of litters in parentheses)

\begin{tabular}{|c|c|c|c|}
\hline & (Control) & $\begin{array}{l}5000 \\
\text { ppm BCF }\end{array}$ & $\begin{array}{l}25000 \\
\text { ppm BCF }\end{array}$ \\
\hline \multicolumn{4}{|l|}{$\begin{array}{l}\text { Unfolding of } \\
\text { pinna: }\end{array}$} \\
\hline Start' & $\begin{array}{r}3.3(3) \\
\pm 0.6\end{array}$ & $3 \cdot 0 \quad(1)$ & $\begin{array}{r}3.5(2) \\
\pm 0.7\end{array}$ \\
\hline Complete $^{2}$ & $\begin{array}{ll} & 4 \cdot 1(12) \\
\pm & 0.3\end{array}$ & $\begin{array}{l}4 \cdot 2(12) \\
\pm \quad 1 \cdot 1\end{array}$ & $\begin{array}{ll} & 3.7(10)^{*} \\
\pm & 0.5\end{array}$ \\
\hline $\begin{array}{l}\text { Hair growth: } \\
\text { Start }\end{array}$ & $\begin{array}{r}6.8(12) \\
+\quad 0.6\end{array}$ & $6.9(11)$ & $6.9(11)$ \\
\hline Complete $^{4}$ & $\begin{array}{l} \pm 0.6 \\
18.8(12) \\
\pm 1.1\end{array}$ & $\begin{array}{l} \pm 1.0 \\
19.0(11) \\
\pm 1.2\end{array}$ & $\begin{array}{l} \pm 1.0 \\
18 \cdot 5(11) \\
\pm 1.0\end{array}$ \\
\hline \multicolumn{4}{|l|}{ Eye opening: } \\
\hline Start & $\begin{array}{r}14.7(11) \\
\pm \quad 0.9\end{array}$ & $\begin{aligned} & 15.0(11) \\
\pm & 0.9\end{aligned}$ & $\begin{array}{r}14.9 \text { (9) } \\
\pm \quad 1.2\end{array}$ \\
\hline Complete $^{6}$ & $\begin{array}{l}16 \cdot 3(12) \\
\pm \quad 1.1\end{array}$ & $\begin{array}{l}16.2(11) \\
\pm \quad 0.8\end{array}$ & $\begin{array}{l}16.3(11) \\
\pm \quad 1.2\end{array}$ \\
\hline \multicolumn{4}{|l|}{ Weaning: } \\
\hline Start ${ }^{5}$ & $\begin{array}{r}22.2 \text { (1) } \\
\pm \quad 1.6\end{array}$ & $\begin{array}{l}21.8(11) \\
\pm \quad 1.6\end{array}$ & $\begin{array}{l}20.9 \cdot(11) \\
\pm \quad 1.0\end{array}$ \\
\hline
\end{tabular}

Age + When pinna detachment was starting in some or all pups.

Age when pinna detachment was complete in all pups.

Age when hair growth was starting in some or all pups.

4 Age when hair growth was complete in all pups.

5 Age when eyes were first seen to be opening in some of the pups.

- Age when eyes were open in all of the pups.

7 Age when some of the pups were first observed eating food

Day of birth = Day 1 .

*Statistically significantly different from the control group mean at the $5 \%$ level ( $t$ test, two sided).

Table 4 BCF reproduction study: number of pups alive at birth and at 27 days postpartum. (Values are means \pm standard deviation with number of litters in parentheses)

\begin{tabular}{|c|c|c|c|c|c|c|c|c|c|}
\hline & \multicolumn{3}{|l|}{ Control } & \multicolumn{3}{|c|}{5000 ppm BCF } & \multicolumn{3}{|c|}{25000 ppm BCF } \\
\hline & $\begin{array}{l}\text { No of } \\
\text { male pups } \\
\text { a litter }\end{array}$ & $\begin{array}{l}\text { No of } \\
\text { female pups } \\
\text { a litter }\end{array}$ & $\begin{array}{l}\text { Males } \\
\text { (males }+ \\
\text { females) }\end{array}$ & $\begin{array}{l}\text { No of } \\
\text { male pups } \\
\text { a litter }\end{array}$ & $\begin{array}{l}\text { No of } \\
\text { female pups } \\
\text { a litter }\end{array}$ & $\begin{array}{l}\text { Males } \\
\text { (males }+ \\
\text { females) }\end{array}$ & $\begin{array}{l}\text { No of } \\
\text { male pups } \\
\text { a litter }\end{array}$ & $\begin{array}{l}\text { No of } \\
\text { female pups } \\
\text { a litter }\end{array}$ & $\begin{array}{l}\text { Males } \\
\text { (males }+ \\
\text { females) }\end{array}$ \\
\hline Day 1 & $\begin{aligned} & 5 \cdot 2(12) \\
\pm & 2 \cdot 6\end{aligned}$ & $\begin{aligned} & 5 \cdot 6(12) \\
\pm & 1 \cdot 9\end{aligned}$ & $\begin{aligned} & 0.46(12) \\
\pm & 0.14\end{aligned}$ & $\begin{aligned} & 5 \cdot 9(12) \\
\pm & 2 \cdot 3\end{aligned}$ & $\begin{aligned} & 5 \cdot 8(12) \\
\pm & 1 \cdot 8\end{aligned}$ & $\begin{aligned} & 0.50(12) \\
\pm & 0 \cdot 14\end{aligned}$ & $\begin{aligned} & 4 \cdot 6(12) \\
\pm & 2 \cdot 4\end{aligned}$ & $\begin{aligned} & 4 \cdot 7(12) \\
\pm & 1 \cdot 6\end{aligned}$ & $\begin{aligned} & 0.47(12) \\
\pm & 0.15\end{aligned}$ \\
\hline Day 27 & $\begin{aligned} & 5 \cdot 0(12) \\
\pm & 2 \cdot 6\end{aligned}$ & $\begin{aligned} & 5.5(12) \\
\pm & 1.9\end{aligned}$ & $\begin{array}{l}0.46(11) \\
\pm 0.14\end{array}$ & $\begin{aligned} & 5.9(11) \\
\pm & 2 \cdot 0\end{aligned}$ & $\begin{aligned} & 5.4(11) \\
\pm & 1.5\end{aligned}$ & $\begin{array}{l}0.52(11) \\
\pm 0.11\end{array}$ & $\begin{aligned} & 4 \cdot 8(11) \\
\pm & 2 \cdot 1\end{aligned}$ & $\begin{aligned} & 4 \cdot 8(11) \\
\pm & 1 \cdot 5\end{aligned}$ & $\begin{aligned} & 0.48(11) \\
\pm & 0.14\end{aligned}$ \\
\hline
\end{tabular}

Table 5 BCF reproduction study: lactation index and per cent mortality. (Values are means \pm standard deviation with number of litters in parentheses)

\begin{tabular}{|c|c|c|c|c|c|c|}
\hline & $\begin{array}{l}\text { No born } \\
\text { alive }\end{array}$ & $\begin{array}{l}\text { No alive } \\
\text { at } 4 \text { days }\end{array}$ & $\begin{array}{l}\text { No alive } \\
\text { at } 28 \text { days }\end{array}$ & $\begin{array}{l}\text { No dead* } \\
\text { at } 28 \text { days }\end{array}$ & $\begin{array}{l}\text { Lactationt } \\
\text { index }\end{array}$ & Mortality \%‡ \\
\hline Control & $\begin{array}{r}10.8(12) \\
\pm \quad 3.6\end{array}$ & $\begin{array}{r}10.6(12) \\
\pm \quad 3.6\end{array}$ & $\begin{array}{r}10.5(12) \\
\pm 3.6\end{array}$ & $\begin{array}{r}0.25 \text { (12) } \\
\pm 0.45\end{array}$ & $\begin{array}{r}0.99 \text { (12) } \\
\pm 0.03\end{array}$ & $\begin{array}{r}2 \cdot 2(12) \\
\pm \quad 4 \cdot 0\end{array}$ \\
\hline 5000 ppm BCF & $\begin{array}{l}11 \cdot 7(12) \\
\pm \quad 2 \cdot 2 \\
11 \cdot 7 \S(11) \\
\pm 2 \cdot 3\end{array}$ & $\begin{array}{l}11.6(12) \\
\pm 2.2 \\
11 \cdot 7 \S(11) \\
\pm 2.3\end{array}$ & $\begin{array}{l}10 \cdot 3(12) \\
\pm 4 \cdot 0 \\
11 \cdot 3 \S(11) \\
\pm 2 \cdot 5\end{array}$ & $\begin{aligned} & 1.33 \text { (12) } \\
& \pm 3.26 \\
& 0.45 \$(11) \\
& \pm \\
& \pm 1.21\end{aligned}$ & $\begin{array}{l}0.88 \text { (12) } \\
0.29 \\
0.96 \$(11) \\
\pm 0.10\end{array}$ & $\begin{aligned} & 11.7 \\
& \pm 29.4 \\
& 3.7 \S(12) \\
& \pm 10.1\end{aligned}$ \\
\hline 25000 ppm BCF & $\begin{array}{l}\quad 9 \cdot 3(12) \\
\pm \\
3 \cdot 2 \\
9 \cdot 7 \|(11) \\
\pm \\
2 \cdot 9\end{array}$ & $\begin{aligned} & 9 \cdot 1(12) \\
\pm & 3 \cdot 3 \\
& 9 \cdot 6 \|(11) \\
\pm & 2 \cdot 8\end{aligned}$ & $\begin{aligned} & 8 \cdot 8(12) \\
\pm & 3 \cdot 8 \\
& 9 \cdot 6 \|(11) \\
\pm & 2 \cdot 8\end{aligned}$ & $\begin{aligned} & 0.42(12) \\
\pm 1.16 & \\
& 0.09 \\
\pm & 0.30\end{aligned}$ & $\begin{aligned} & 0.92 \\
& \pm 0.29 \\
& 1.00 \|(12) \\
& \pm 0\end{aligned}$ & $\begin{aligned} & 9 \cdot 0(12) \\
\pm & 28 \cdot 8 \\
& 0 \cdot 7 \|(11) \\
\pm & 2 \cdot 3\end{aligned}$ \\
\hline
\end{tabular}

* Excluding stillborns.

+ Number of pups alive at 28 days - number alive at four days.

$\ddagger$ Number of dead pups at 28 days number born alive $\times 100$.

Excluding one dam with impaired lactation (all pups dead by day 8 ).

WExcluding one dam with impaired lactation (all pups dead by day 5). 
Table 7 BCF reproduction study: intergroup comparison of litter data (subgroup B)

\begin{tabular}{|c|c|c|c|}
\hline & \multicolumn{3}{|c|}{ Exposure concentration of $B C F$} \\
\hline & 0 ppm (Control) & $5000 \mathrm{ppm}$ & $2500 \mathrm{ppm}$ \\
\hline \multirow[b]{2}{*}{$\begin{array}{l}\text { Mean No of implantations } \\
\text { Embryonic deaths: } \\
\text { No and \%, early } \\
\text { No and \%, late } \\
\text { Mean No of fetuses } \\
\text { Mean No of corpora lutea } \\
\text { Preimplantation loss \% } \\
\text { Postimplantation loss \% } \\
\text { No of pregnant females } \\
\text { No of non-pregnant females }\end{array}$} & $13.38 \pm 1.77$ & $11.00 \pm 3.55$ & $11.88 * \pm 1.36$ \\
\hline & $\begin{array}{cc}2 & 1.87 \% \\
0 & 0 \% \\
13.13 & \pm 1.81 \\
14.00 & \pm 1.07 \\
6.34 & \pm 8.49 \\
1.79 & \pm 5.05 \\
8 & \\
4 & \end{array}$ & $\begin{array}{cc}3 & 3.41 \% \\
0 & 0 \% \\
10.63 \pm 3.66 \\
13.00 \pm 0.926 \\
16.69 \pm 23.78 \\
4.91 \pm 9.53 \\
8 \\
4\end{array}$ & $\begin{array}{ll}5 & 5 \% \\
1 & 1 \% \\
11 \cdot 13 * & \pm 1.73 \\
12.88 & \pm 1.36 \\
8.33 & \pm 8.47 \\
5.70 & \pm 6.79 \\
8 & \\
4 & \end{array}$ \\
\hline
\end{tabular}

*Statistically significantly different from the control group at the $5 \%$ level ( $t$ tested, one sided).

Discussion

An increased awareness of the potential for chemicals to perturb the process of reproduction has meant that this aspect of toxicology has received greater emphasis in recent years. For this reason it was decided to assess the capacity of BCF to cause reproductive disturbances in laboratory animals. The effect of acute single exposure to $\mathrm{BCF}$, such as may be experienced during fire fighting, is primarily on the central nervous system $^{1}$ and there are no indications of any effects that might be expected to produce reproductive toxicity. Nevertheless, it was thought prudent to examine BCF using study designs which would detect any significant effects. The studies chosen investigate three important aspects of the reproductive process: the fertility of adults exposed to the compound, the effect of the compound on the developing offspring in utero, and the postnatal development of the offspring.

The teratogenicity phase of the investigation showed that BCF is not teratogenic in the rat even at $50000 \mathrm{ppm}$ which had a definite, although minor, effect on the dams as shown by a reduction in bodyweight gain during the exposure period. There were no indications of any adverse effects on the developing fetuses, the reduced fetal weight in the $10000 \mathrm{ppm}$ BCF group being considered unrelated to treatment in the absence of any effect at $50000 \mathrm{ppm}$.

The reproductive toxicity phase of the investigation showed no effect on adult fertility in either sex. There was a minor reduction on the number of fetuses in the dams exposed to $25000 \mathrm{ppm}$ for two weeks before mating and during the mating phase. The observation was not repeated in the females subsequently exposed to BCF during gestation and this must limit the significance of the finding. The pups gained weight and developed normally, with no effect on survival indicating that BCF does not have any adverse effect on reproduction or the subsequent growth and development of the offspring even with extended exposures to high levels.

Thus BCF must be considered to have no significant reproductive toxicity potential in an occupational setting.

\section{References}

1 Beck PS, Clark DG, Tinston DJ. The pharmacological actions of bromochlorodifluoromethane (BCF). Toxicol Appl Pharmacol 1973;24:20-9.

2 Doe JE, Tinston DJ. Novel chambers for long term inhalation studies. In: Leong BHJ, ed. Proceedings of the inhalation toxicology and technology symposium, Kalamazoo, 1980. Michigan: Ann Arbor Science, 1981:776-88. 